\title{
The Discovery and Exploratory Development of Maraviroc (UK-427,857): A Novel CCR5 Antagonist for the Treatment of HIV CA Hitchcock*
}

Address: Pfizer Global Research and Development, Sandwich Laboratories, Sandwich CT13 9NJ, UK

* Corresponding author ‡Presenting author

from 2005 International Meeting of The Institute of Human Virology

Baltimore, USA, 29 August - 2 September 2005

Published: 8 December 2005

Retrovirology 2005, 2(SuppI I):SII doi:I0.II86/I742-4690-2-SI-SII

Maraviroc is a novel CCR5 antagonist and is the most advanced clinical candidate in Pfizer's CCR5 discovery and development programme. It is exquisitely selective for the CCR 5 receptor and demonstrates potent activity in vitro against both lab-adapted and primary clinical HIV isolates spanning all of the clades, including viruses that are resistant to current classes of HIV agents. Maraviroc has been evaluated in $>400$ volunteers and in $66 \mathrm{HIV}$ patients where it is well tolerated at doses in excess of those required to block the CCR5 receptor and those providing free drug levels above the in vitro concentrations for potent antiviral activity. Consistent with this, Maraviroc has demonstrated encouraging short-term (10 day), single agent efficacy as measured by reductions in viral loads in asymptomatic HIV patients; doses of $300 \mathrm{mg}$ QD and 300 mg BID resulted in mean maximum HIV RNA reductions of $1.60 \log _{10}$ and $1.84 \log _{10}$, respectively. Studies both with CYP 3A4 inhibitors and inducers have demonstrated that Maraviroc will have manageable drug interactions when used in the setting of HIV patients receiving HAART. In summary, Maraviroc has potency, pharmacokinetic and toleration profiles that merit its further evaluation as a new therapy for patients with HIV/AIDS. 